\title{
Mice with ablated adult brain neurogenesis are not impaired in antidepressant response to chronic fluoxetine
}

\author{
Paulina Jedynak a, Tomasz Kos ${ }^{\text {b }}$, Carmen Sandi ${ }^{\mathrm{c}}$, Leszek Kaczmarek ${ }^{\mathrm{a}}$, \\ Robert K. Filipkowski a, d, e, * \\ ${ }^{a}$ Laboratory of Neurobiology, Nencki Institute of Experimental Biology, Polish Academy of Sciences, 02-093 Warsaw, Poland \\ b Department of Behavioral Neuroscience \& Drug Development, Institute of Pharmacology, Polish Academy of Sciences, 31-343 Krakow, Poland \\ ${ }^{\mathrm{c}}$ Laboratory of Behavioral Genetics, Brain Mind Institute, Ecole Polytechnique Federale de Lausanne (EPFL), CH-1015 Lausanne, Switzerland \\ d Department of Biological Psychology, University of Finance and Management in Warsaw, 01-030 Warsaw, Poland \\ e Behavior and Metabolism Research Laboratory, Mossakowski Medical Research Centre, Polish Academy of Sciences, 02-106 Warsaw, Poland
}

\section{A R T I C L E I N F O}

\section{Article history:}

Received 14 January 2014

Received in revised form

29 April 2014

Accepted 13 May 2014

\section{Keywords:}

Adult neurogenesis

Dentate gyrus

Depression-like behavior

Fluoxetine

Stress

\begin{abstract}
A B S T R A C $T$
The neurogenesis hypothesis of major depression has two main facets. One states that the illness results from decreased neurogenesis while the other claims that the very functioning of antidepressants depends on increased neurogenesis. In order to verify the latter, we have used cyclin D2 knockout mice (cD2 KO mice), known to have virtually no adult brain neurogenesis, and we demonstrate that these mice successfully respond to chronic fluoxetine. After unpredictable chronic mild stress, mutant mice showed depression-like behavior in forced swim test, which was eliminated with chronic fluoxetine treatment, despite its lack of impact on adult hippocampal neurogenesis in cD2 KO mice. Our results suggest that new neurons are not indispensable for the action of antidepressants such as fluoxetine. Using forced swim test and tail suspension test, we also did not observe depression-like behavior in control $\mathrm{cD} 2 \mathrm{KO}$ mice, which argues against the link between decreased adult brain neurogenesis and major depression.
\end{abstract}

() 2014 Elsevier Ltd. All rights reserved.

\section{Introduction}

Major depression is a common mental disorder, however, its pathophysiology remains poorly understood. Stress, the main cause of depression, most severely affects hippocampal formation (McEwen et al., 2012), while hippocampal subgranular zone (SGZ) of the dentate gyrus (DG) is a region where new neurons originate throughout mammalian adulthood. Moreover, stress leads to significant decrease of adult neurogenesis (for review see WarnerSchmidt and Duman, 2006), implicating a role of this decrease in the onset of depression-like symptoms, however, this notion remains controversial (for reviews see Sahay and Hen, 2007; Balu and Lucki, 2009; Eisch and Petrik, 2012).

Hippocampal neurogenesis has also been proposed as a crucial process involved in the therapeutic efficacy of chronic antidepressants (ADs) treatment (e.g., Santarelli et al., 2003). This hypothesis

\footnotetext{
* Corresponding author. Department of Biological Psychology, University of Finance and Management in Warsaw, 55, Pawia St., 01-030 Warsaw, Poland. Tel.: +48 668403448; fax: +48 225365412.

E-mail_addresses:_filipkowski@vizja.pl,_rfilipkowski@imdik.pan.pl
} (R.K. Filipkowski). is supported by the fact that recovery from depression requires several weeks - the timescale that overlaps with the time-course of $\mathrm{AD}$-stimulated neurogenesis and is also paralleled with the time needed for differentiation and incorporation of newborn neurons into existing neuronal hippocampal networks (Sahay and Hen, 2007; Balu and Lucki, 2009). Moreover, majority of the antidepressant approaches elevate neurogenesis by increasing proliferation rate and/or by enhancing newborn cells survival (for review see Samuels and Hen, 2011). Indeed, it was shown that animals with blocked adult neurogenesis do not recover from depressionlike behavior when chronically administered with ADs (Santarelli et al., 2003; Surget et al., 2008; David et al., 2009; Onksen et al., 2011; Perera et al., 2011). However, recent studies suggest both neurogenesis-dependent and independent mechanisms underlying ADs action, as more studies show none or only partial effect of reducing neurogenesis on restoration of behavioral homeostasis by ADs (Meshi et al., 2006; David et al., 2007; Holick et al., 2008; Surget et al., 2008; Bessa et al., 2009a; David et al., 2009; Singer et al., 2009; Nollet et al., 2012).

Herein, we have employed cyclin D2 knockout (cD2 KO) mice showing lack of adult brain neurogenesis. We showed before that mice with mutated cyclin D2 gene display largely impaired 
proliferation of neuronal precursors in SGZ (Kowalczyk et al., 2004; Jaholkowski et al., 2009; Jedynak et al., 2012). Notably, cyclin D2 mutant mice show deficits in some hippocampal-dependent behaviors (Jedynak et al., 2012), but not in learning in general (Jaholkowski et al., 2009; Jedynak et al., 2012; Urbach et al., 2013), while selected cognitive functions are impaired (Ben Abdallah et al., 2013; Garthe et al., 2014).

In the present study, we set out to test the reaction of $\mathrm{cD} 2 \mathrm{KO}$ mice to chronic fluoxetine administration following chronic stress. We have chosen unpredictable chronic mild stress (UCMS) as known to cause ADs-reversible behavioral changes in rodents that parallel symptoms of major depression (Willner, 2005). This paradigm was also used in the original study suggesting a causal link between ADs efficacy and adult neurogenesis (Santarelli et al., 2003). Also, we have chosen forced swim test (FST) as one of the most widely used test of ADs action (Petit-Demouliere et al., 2005; Krishnan and Nestler, 2011), which results in reduced immobility following their acute (Porsolt et al., 1977a, 1977b) and chronic delivery (e.g., Detke et al., 1997; Dulawa et al., 2004; Holick et al., 2008; Lin and Wang, 2014) as well as in reversal of increased immobility following UCMS (Willner, 2005). Finally, we have used fluoxetine as most commonly used AD in adult neurogenesisdepression studies, e.g., in the original Santarelli et al. (2003) publication.

\section{Materials and methods}

\subsection{General experimental design}

A cohort of animals (cD2 KO, $n=30$; WT, $n=30$ ) was subjected to unpredictable chronic mild stress (UCMS) and chronically administered with fluoxetine. When stress and fluoxetine administration ended, all animals were tested behaviorally in the forced swim test (FST) and cell proliferation was assessed in DG using bromodeoxyuridine (BrdU) injection and immunocytochemistry. There was also an open field control test performed $24 \mathrm{~h}$ after the last fluoxetine administration. The overall design of experimental procedures is shown in Fig. 1.

\subsection{Animals}

Cyclin D2 mutant mice (Kowalczyk et al., 2004) were backcrossed into C57BL/6 background over 10 generations and kept as cyclin D2 heterozygotes $(+/-)$. Their homozygous progeny, cD2 KO $(-/-)$ and $\mathrm{WT}(+/+)$ littermates, were used. If not stated otherwise, the animals were kept under a natural light/dark cycle in Plexiglas cages with water and food provided ad libitum. To ensure proper care and use of laboratory animals, the national rules according to the Animal Protection Act, ensured by the permission from the First Warsaw Local Ethics Committee for Animal Experimentation, were strictly followed. The animals were males, 2-4 months old at the beginning of the experiments, with their age carefully matched between WT and cD2 KO mice. Experimenters were always unaware of the genotype of the mice.

\subsection{Unpredictable chronic mild stress (UCMS) and chronic fluoxetine treatment}

The mice ( $\mathrm{cD} 2 \mathrm{KO}, n=29$; WT, $n=29$ ) were divided into three age-matched groups (non-stressed, $n=10$; stressed-vehicle, $n=10$; stressed-fluoxetine, $n=9$ ). UCMS protocol was described before (Bisaz et al., 2011; Bisaz and Sandi, 2012) and used with some modifications. Non-stressed and stressed groups were housed in separated and closed housing racks located in the same room during the duration of the stress procedure. As the experiment started, non-stressed groups were left undisturbed. Stressed groups were exposed to UCMS procedure which consisted of different kinds of stressors: cage tilting, damp sawdust, housing in an empty cage, pairing with another stressed animal, cold room, water or food deprivation, inversion of the light/dark cycle, lights on for a short period of time during the dark phase and switching cages amongst stressed animals. One or two of these stressors were applied daily at different times and following a semi-random schedule. Starting from the beginning of the 4th week of UCMS, stressed mice were given either $10 \mathrm{mg} / \mathrm{kg} /$ day (comp. Santarelli et al., 2003; Bessa et al., 2009a) fluoxetine hydrochloride (Sigma, $\mathrm{PL})$, dissolved in water and prepared freshly before use, or water. The treatment lasted for 3 weeks and was provided via oral gavage (Fine Science Tools Inc., USA). The drug concentration was adjusted weekly from the average body weight of mice to achieve the desired doses.

\subsection{Forced swim test (FST)}

In order to eliminate the effects of acute fluoxetine injections, FST was performed 3 days after the last delivery of the drug. The test was conducted as described by Porsolt et al. (1977a, 1977b) by placing mice into a glass cylinder $(25 \mathrm{~cm}$ height, $10 \mathrm{~cm}$ diameter) containing $1250 \mathrm{ml}$ of water maintained at $24-25{ }^{\circ} \mathrm{C}$. The test lasted for $6 \mathrm{~min}$. Starting from the 3rd minute of the test, mice were rated for immobility defined as the absence of active, escapeoriented behaviors such as swimming, jumping, climbing, or diving. Two animals were tested simultaneously.

\subsection{Tail suspension test (TST)}

TST procedure was previously described (Steru et al., 1985). Mice were suspended by the tail temporarily attached to a metal bar using adhesive scotch tape. Total duration of immobility was counted during a 6 min test. Three animals were tested simultaneously. One WT animal climbing its tail was excluded from the experiment.

\subsection{Open field}

The test was performed $24 \mathrm{~h}$ after the termination of UCMS and fluoxetine treatment, it was done as described previously (Jedynak et al., 2012). The apparatus was a wooden floor $(50 \mathrm{~cm} \times 50 \mathrm{~cm})$ surrounded by $34 \mathrm{~cm}$ high walls. Gray walls and floor as well as

UCMS

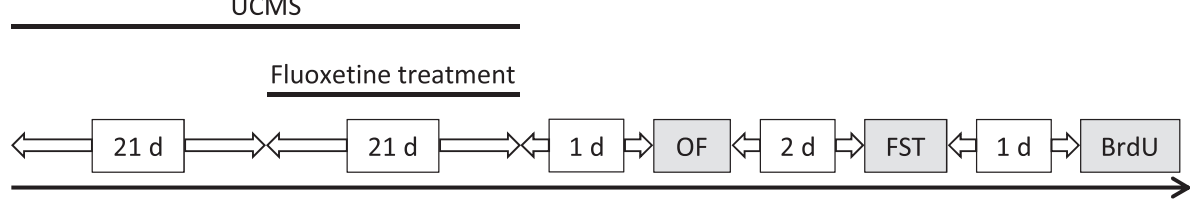

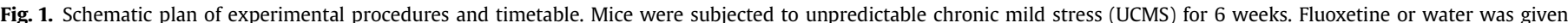

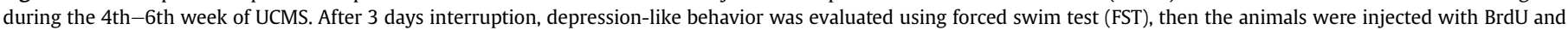
perfused for immunocytochemistry. Control open field (OF) was performed $24 \mathrm{~h}$ after the last fluoxetine treatment. 
normal room illumination were used to prevent suppression of activity and exploration behaviors by the anxiety response. Animals' behavior was monitored by a video camera placed above the center of the apparatus. Mice were put individually in one corner of the open field facing the wall and were allowed to explore freely for 5 min. Four open fields were employed to test four animals simultaneously. The floor of the apparatus was cleaned with $5 \%$ ethanol after each session. Data were analyzed using EthoVision 8.0 system (Noldus Information Technology, The Netherlands), and the total distance moved was acquired.

\subsection{Perfusion and sectioning}

To assess changes in cell proliferation in DG after chronic stress and chronic fluoxetine treatment, $24 \mathrm{~h}$ after completion of behavioral testing, mice were injected once with bromodeoxyuridine (150 mg/kg, i.p., Sigma, prepared in PBS, pH 7.4). Two hours after injection, mice were anesthetized and killed by cardiac perfusion (4\% paraformaldehyde). Brains were then post-fixed overnight in $4 \%$ paraformaldehyde at $4{ }^{\circ} \mathrm{C}$ then transferred into a 30\% sucrose/ $0.1 \%$ sodium azide cryoprotectant until the brains sank. Brains were embedded in tissue freezing medium, and sectioned using a cryostat to cut $50 \mu \mathrm{m}$ coronal sections. Six to eight sections from each brain were analyzed, -1.28 to $-2.75 \mathrm{~mm}$ from the bregma.

\subsection{Bromodeoxyuridine immunohistochemistry}

BrdU detection was performed as described elsewhere (Kowalczyk et al., 2004), with modifications. Free-floating sections were rinsed with PBS, then incubated in $2 \mathrm{~N} \mathrm{HCl}$ for 30 min at $37^{\circ} \mathrm{C}$ and neutralized by immersing in $0.1 \mathrm{M}$ sodium tetra-borate buffer for $10 \mathrm{~min}$. Sections were rinsed again in PBS and PBS-TX containing $0.01 \%$ Triton $\mathrm{x}-100$ and then blocked with $10 \%$ normal donkey serum (NDS) for $1 \mathrm{~h}$. Primary antibody (sheep anti-BrdU in 1\% NDS, 1:500, Abcam, UK) was applied with overnight incubation. The following day, sections were washed of primary antibody, and incubated for $1 \mathrm{~h}$ with Alexa 488 secondary antibody (donkey antisheep in PBS, 1:500, Abcam, UK). Sections were mounted with Fluoromount-G containing DAPI nuclear marker (Southern Biotech., USA) and coverslipped. The mean numbers of BrdU+ cells per section in SGZ of DG were calculated, using $\times 40$ magnification of an Olympus fluorescent microscope.

\subsection{Data analysis}

Statistical analyses were performed with GraphPad Prism 5.00 (GraphPad Software, USA). Data are reported as mean + SEM (if distributed normally) or as median with inter-quartile range (IQR), minimum and maximum values (when not distributed normally). When possible, data were submitted to ANOVA or a nonparametrical Mann-Whitney test was used (indicated in the text).

\section{Results}

\subsection{Mutant $\mathrm{CD} 2 \mathrm{KO}$ mice did not show increased immobility}

Basal depression-like behavior, behavioral response to UCMS as well as chronic fluoxetine treatment were assessed in FST (Fig. 2A). Two-way ANOVA of no stress and stress-vehicle groups revealed effect of both the stress $(p<0.001, F(1,39)=24.0)$ and genotype $(p<0.01, F(1,39)=9.3)$. The latter effect was confirmed by one-way ANOVA: cD2 KO mice showed decreased time [\%] spent immobile compared to WT animals (cD2 KO mice, $38.6 \pm 3.4$; WT mice, $51.5 \pm 3.5 ; F(1,19)=6.9, p<0.05)$. We further confirmed this observation by testing immobility in FST and tail suspension test
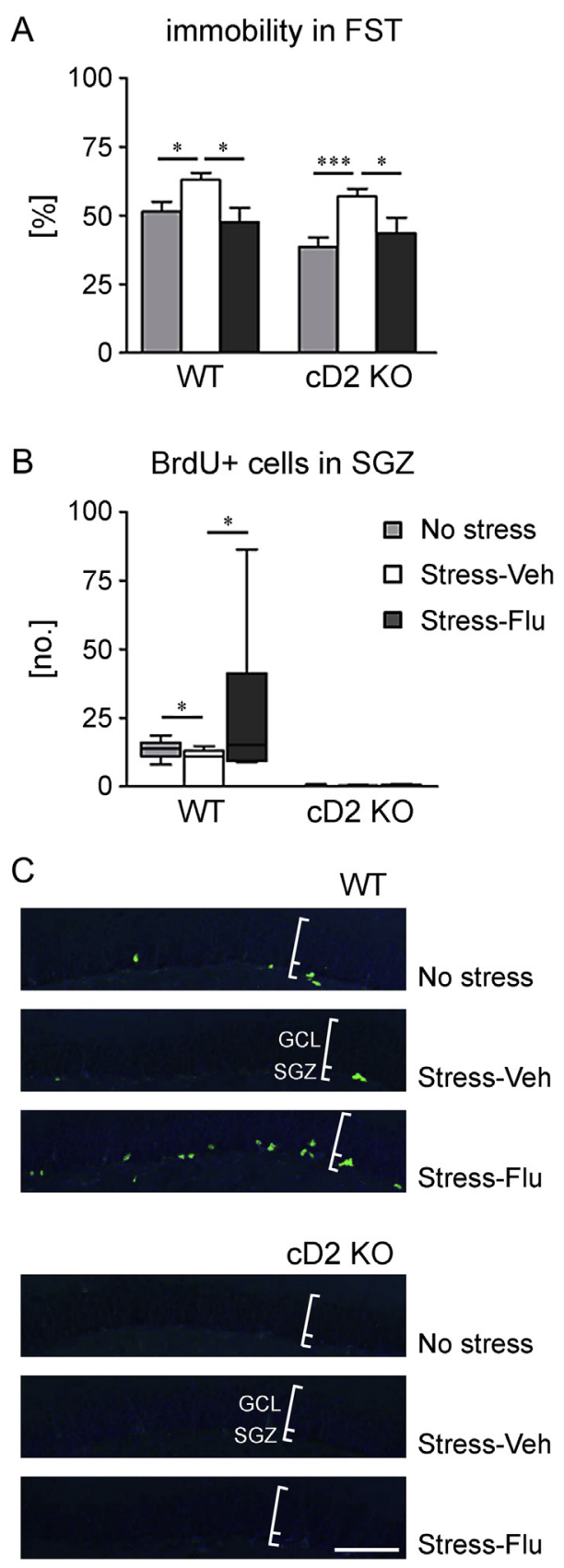

Fig. 2. Behavioral and cellular response to chronic stress and fluoxetine treatment in cD2 KO and WT mice. In FST (A), behavioral effects of chronic stress (Stress-Veh) were manifested by increased immobility time in both CD2 KO and WT animals. Chronic fluoxetine treatment (Stress-Flu) abolished this difference independently of the genotype as both CD2 KO and WT animals administered with fluoxetine showed immobility time similar to non-stressed mice. In WT mice, chronic stress caused significant decrease in the number of BrdU+ cells in the SGZ of the hippocampal formation (B, C). The effect was prevented by chronic fluoxetine treatment. There was no effect on neurogenesis in $\mathrm{CD} 2 \mathrm{KO}$ animals in any conditions. The mean number of new cells per slice (B) is shown along with representative parts of DG granule cell layer (GCL) with the SGZ and GCL areas indicated (C). Data shown represent the means + SEM (A) or medians with inter-quartile range, minimum and maximum values (B). ${ }^{*} p<0.05 ;{ }^{* * *} p<0.001$; scale bar, $100 \mu \mathrm{m}$.

(TST) using separate groups of mice (not shown). In both tests, nonstressed, naive cD2 KO mice presented reduced immobility when compared to WT mice. The decrease in immobility duration [\%] was observed in FST (cD2 KO mice, $35.5 \pm 6.4, n=9$; WT mice, $53.7 \pm 3.1$, $n=11 ; F(1,19)=3.5, p<0.05$, one-way ANOVA) and TST (cD2 KO 
mice, $25.7 \pm 1.6, n=6$; WT mice, $37.3 \pm 3.9, n=6, F(1,11)=7.5$, $p<0.05$, one-way ANOVA).

\subsection{Mutant mice show depression-like behaviors after stress and respond to fluoxetine treatment}

The effect of stress was also confirmed as UCMS resulted in an increase of immobility time [\%] in both $\mathrm{CD} 2 \mathrm{KO}(57.0 \pm 2.7, p<0.001$, $F(1,19)=17.5)$ as well as WT mice $(63.0 \pm 2.5, p<0.05, F(1,19)=7.1$, one-way ANOVA). Interestingly, CD2 KO and WT animals responded also equally well to chronic fluoxetine administration. Two-way ANOVA revealed effect of fluoxetine treatment $(p<0.01$, $F(1,37)=12.0)$ but no effect of genotype. Both groups of fluoxetinetreated mice showed reduced immobility time [\%] compared with vehicle-treated animals (cD2 KO mice, $43.6 \pm 5.6, p<0.05$, $F(1,17)=4.9$; WT mice, $47.6 \pm 5.2, p<0.05, F(1,17)=7.5$; one-way ANOVA).

Overall influence of fluoxetine treatment on animals activity was examined in the open field. One-way ANOVA analysis of the total distance moved reveal no effect of treatment in any group (cD2 KO mice, $F(2,28)=0.2, p=0.8$; WT mice, $F(2,28)=1.35$, $p=0.3$ ). Importantly, chronic fluoxetine did not increase the total distance $[\mathrm{m}]$ in $\mathrm{CD} 2 \mathrm{KO}$ mice $(26.5 \pm 2,0)$ when compared to vehicle-treated group $(28.1 \pm 1.9)$ and non-treated mice $(28.5 \pm 2.0)$. Fluoxetine treatment had a similar effect also in WT control animals $(23.6 \pm 1.7 ; 26.3 \pm 2.1 ; 23.5 \pm 1.4$; respectively $)$.

\subsection{Neurogenic effect of chronic fluoxetine administration observed only in control animals}

Detailed stereological study of adult neurogenesis in naive cD2 KO mice in comparison to WT littermates was described elsewhere (Jaholkowski et al., 2009). In the present study, we confirmed that non-stressed cD2 KO mice showed almost complete lack of BrdU+ cells in SGZ of DG $(0.0 ; 0.0-0.2$, IQR $)$ compared to WT mice (13.9; 13.2-15.5, IQR; $p<0.001$, Mann-Whitney, $\mathrm{U}=0$; Fig. 2BC). Chronic stress decreased numbers of BrdU+ cells in WT animals (11.0; 0.2-12.6, IQR), $p<0.05$, Mann-Whitney, $U=18$ ) when compared to non-stressed ones, and had no effect in cD2 KO mice (0.1; 0.0-0.3, IQR; $p>0.05$, Mann-Whitney). Chronic fluoxetine treatment caused significant increase of the number of proliferating cells in SGZ of WT mice when compared to vehicle-treated WT animals (15.2; 9.6-31.0, IQR, $p<0.05$, Mann-Whitney, $U=17)$. There was no neurogenic effect of chronic fluoxetine administration in $\mathrm{cD} 2 \mathrm{KO}$ animals $(0.3 ; 0.0-0.5$, IQR; $p>0.05$, Mann-Whitney).

\section{Discussion}

Despite numerous publications, the exact function of new neurons in the etiology and treatment of mood disorders remains elusive. In the present study, we used cD2 KO mice with ablated adult neurogenesis to investigate the role of this process in ADs action. We have found that fluoxetine treatment was efficient in the FST independently of the presence of newborn cells in the SGZ of mice. These data suggest that neurogenesis-independent mechanisms underlie, at least in some specific circumstances, the behavioral effect of fluoxetine, and that the increase in adult neurogenesis observed in WT animals is rather an epiphenomenon not necessary for the drug's direct effect.

There is limited direct evidence supporting reduced hippocampal neurogenesis in the pathophysiology of depression. Although reduced neurogenesis and depression-like phenotypes arise in tandem following various chronic stressors (e.g., Surget et al., 2008; Bessa et al., 2009a), a requirement for reduced neurogenesis in the development of a depression-like phenotype has apparently been demonstrated only by Conboy et al. (2011). In our hands, however, cD2 KO mice, devoid of adult brain neurogenesis, did not show increased immobility in FST and TST tests. On the contrary, the immobility was reduced in these mice. We have observed before $\mathrm{CD} 2 \mathrm{KO}$ animals to be more active and explorative (Jedynak et al., 2012; Ben Abdallah et al., 2013). Please note, we were still able to increase immobility in mutant mice by UCMS and again reduce it through chronic fluoxetine treatment. The latter effect was apparently not a false positive result of an overall increase in mobility since chronic fluoxetine had no effect on the total distance moved in the open field.

Numerous observations suggest the role of adult brain neurogenesis in the treatment of depression-like behaviors (see Introduction). Importantly, in cD2 KO mice, we did not observe any increase in cell proliferation in the SGZ following chronic fluoxetine administration. It can be explained by the lack of cells targeted by fluoxetine or their proliferation impairment, as it is not clear whether the disruption of cyclin D2 gene causes developmental ablation of amplifying neural progenitors or their inability for divisions. The fact that suppression of depression-like symptoms occurred in both WT and cD2 KO mice, independently of the presence or lack of adult hippocampal neurogenesis points out a neurogenesis-independent mechanism behind chronic fluoxetine action. As concluded before by Bessa et al. (2009a), although temporal coincidence in the occurrence of impaired neurogenesis and depression-like symptoms and their reversal by ADs treatment are evident, a direct cause-effect relationship between neurogenesis and the alleviation of signs of depression-like behavior is missing. It was proposed that ADs, irrespectively of their mechanisms of action, trigger neuronal remodeling and synaptic plasticity and the increase in adult neurogenesis is rather an epiphenomenon (Bessa et al., 2009a). This notion is supported by recent finding that increase of adult hippocampal neurogenesis causes neither an anxiolytic nor antidepressant behavioral effect (Udo et al., 2008; Sahay et al., 2011). There is also accumulating evidence that other brain regions including amygdala, nucleus accumbens, or cingulate cortex are also involved in $\mathrm{AD}$-like activity. It is possible as well that adult neurogenesis outside of the hippocampus may play a role in the effects of fluoxetine (Kokoeva et al., 2007). Nevertheless, our study supports a hippocampal neurogenesis-independent mechanism of fluoxetine-mediated behavioral effect.

It was suggested that fluoxetine acts independently of neurogenesis especially when tested by FST and TST, while other tests, e.g., novelty-suppressed feeding (NSF) and novelty-induced hypophagia (NIH), are neurogenesis-dependent. However, this quality of FST and TST, when compared to other set of tests, was demonstrated only by one group, following rarely-used chronic corticosterone treatment (David et al., 2009; Mendez-David et al., 2014). Moreover, the treatment had no basal effect as corticosterone injections did not increase the immobility levels measured by FST (David et al., 2009, Fig. 1D) and TST (Mendez-David et al., 2014; Fig. 3P) in control mice, nor were acute vs. chronic effects of fluoxetine-delivery investigated. Furthermore, it is known that glucocorticoids affects food intake (e.g., Solano and Jacobson, 1999) which is a crucial element of most of the postulated neurogenesisdependent tests, i.e., NSF, NIH. Also, using parallel FST and NSF protocols, the same group showed that behavioral effects of chronic fluoxetine in $\mathrm{BALB} / \mathrm{cJ}$ mice do not require adult neurogenesis (Holick et al., 2008). Finally, Bessa et al. (2009a) demonstrated that reduction of adult neurogenesis in rats did not change the effectiveness of several ADs in both FST and sucrose preference test, while their efficacy was blocked in NSF. This observation added to the notion that NSF shows anxiolytic rather than antidepressant properties of investigated drugs (discussed in Bessa et al., 2009a; 
Bessa et al., 2009b; David et al., 2009). Finally, the prevailing perception in the literature, suggests FST to be a proper test to show neurogenesis-dependence of antidepressant efficacy. Several groups described X-irradiation to block antidepressant effects using FST (e.g., Zhu et al., 2010; Garza et al., 2012) while correlative observations linking antidepressant treatment effects in FST and adult hippocampal neurogenesis have been reported numerous times (Liu et al., 2008; Silva et al., 2008; Schmidt and Duman, 2010; Wainwright et al., 2011; Wang et al., 2011; Jiang et al., 2012; Lin and Wang, 2014; Lu et al., 2014).

Our experiment was designed to investigate solely the results of chronic fluoxetine treatment, since antidepressant effects of the drug can be observed following acute or short term use, e.g., when administered 30 min before FST testing in mice (Holick et al., 2008). Therefore, 3 days interval between the last fluoxetine delivery and FST testing was introduced (Fig. 1) while even 1 day interval is frequently used and considered sufficient to eliminate short-term fluoxetine effects in rodents (e.g., Castro et al., 2010). On the other hand, fluoxetine-induced adult-born neurons require weeks to become fully functional (Sahay and Hen, 2007), therefore we used 3 weeks plus 3 days between the introduction of the and FST testing. Three weeks of fluoxetine treatment of mice were shown before to have behavioral effects apparently dependent on adult brain neurogenesis (Santarelli et al., 2003; Surget et al., 2008; David et al., 2009). It was also shown that FST model provides a valid assessment of chronic, and not acute, fluoxetine action in mice (Holick et al., 2008).

Finally, we realize that our model and, hence, our findings have limitations. Mutant CD2 KO mice are constitutive knockout animals with the phenotype not limited to adult brain neurogenesis but also including reduction in size of several brain structures. There is also possibility of compensatory mechanisms to operate in these mice (discussed in Jaholkowski et al., 2009), however, the model is recognized as valuable and adequate tool in the field (for review see Frankland, 2013). The results obtained with the use of cD2 KO mice suggested the lack of relevance of adult brain neurogenesis for learning and memory in general (Jaholkowski et al., 2009) - a view which is now widely accepted (e.g., Sahay et al., 2011) - while suggested and/or confirmed the role of this process in particular aspects of learning (Ben Abdallah et al., 2013), smell detection (Jaholkowski et al., 2009), species-typical behaviors (Jedynak et al., 2012), and alcohol consumption (Jaholkowski et al., 2011). Most of transgenic models with manipulated adult hippocampal neurogenesis, in contrast to our model, do not achieve complete ablation of newborn neurons. In case of depression etiology and treatment studies, partial suppression of adult neurogenesis can be considered as an advantage, as such model has the potential to imitate the rate of neurogenesis during aging in humans, which is reduced only partially (Balu and Lucki, 2009). On the other hand, the complete reduction of neurogenesis may prevent compensative effects from residual newborn neurons and potential intra- and inter-group variability. In conclusion, our studies do not support the role of adult neurogenesis in pharmacological treatment of depression with fluoxetine as well as put in question a strategy to search for novel therapeutic approaches aiming at cell-cycle stimulation and induction of adult neurogenesis (comp. Patricio et al., 2013).

\section{Conflict of interest}

All authors declare that they have no conflicts of interest.

\section{Contributors}

LK, RKF, and PJ designed the study. TK and CS introduced FST and UCMS techniques, respectively, into the laboratory. PJ performed the experiments and undertook the statistical analysis. PJ and RKF wrote the manuscript and prepared the figures. All authors contributed to and have approved the final manuscript.

\section{Role of funding sources}

This work was supported by the European Union structural funds Innovative Economy Operational Program, project no. POIG.01.01.02-00-109/09 and by statutory funds of the University of Finance and Management in Warsaw. PJ was supported by FEBS Short-Term Collaborative Experimental Scholarship for Central \& Eastern Europe. The funding sources had no role in the study design; collection, analysis or interpretation of data; writing of the report; or decision to submit the paper for publication.

\section{Acknowledgments}

None.

\section{References}

Balu DT, Lucki I. Adult hippocampal neurogenesis: regulation, functional implications, and contribution to disease pathology. Neurosci Biobehav Rev 2009;33: 232-52.

Ben Abdallah NM, Filipkowski RK, Pruschy M, Jaholkowski P, Winkler J, Kaczmarek L, et al. Impaired long-term memory retention: common denominator for acutely or genetically reduced hippocampal neurogenesis in adult mice. Behav Brain Res 2013;252:275-86.

Bessa JM, Ferreira D, Melo I, Marques F, Cerqueira JJ, Palha JA, et al. The moodimproving actions of antidepressants do not depend on neurogenesis but are associated with neuronal remodeling. Mol Psychiatr 2009a;14. 764-73,739.

Bessa JM, Mesquita AR, Oliveira M, Pego JM, Cerqueira JJ, Palha JA, et al. A transdimensional approach to the behavioral aspects of depression. Front Behav Neurosci 2009b;3(1).

Bisaz R, Sandi C. Vulnerability of conditional NCAM-deficient mice to develop stress-induced behavioral alterations. Stress 2012;15:195-206.

Bisaz R, Schachner M, Sandi C. Causal evidence for the involvement of the neural cell adhesion molecule, NCAM, in chronic stress-induced cognitive impairments. Hippocampus 2011:21:56-71.

Castro JE, Varea E, Marquez C, Cordero MI, Poirier G, Sandi C. Role of the amygdala in antidepressant effects on hippocampal cell proliferation and survival and on depression-like behavior in the rat. PloS One 2010;5:e8618.

Conboy L, Varea E, Castro JE, Sakouhi-Ouertatani H, Calandra T, Lashuel HA, et al. Macrophage migration inhibitory factor is critically involved in basal and fluoxetine-stimulated adult hippocampal cell proliferation and in anxiety depression, and memory-related behaviors. Mol Psychiatr 2011;16:533-47.

David DJ, Klemenhagen KC, Holick KA, Saxe MD, Mendez I, Santarelli L, et al. Efficacy of the MCHR1 antagonist N-[3-(1-\{[4-(3,4-difluorophenoxy)phenyl]methyl\}(4piperidyl))-4-methylphenyl]-2-m ethylpropanamide (SNAP 94847) in mouse models of anxiety and depression following acute and chronic administration is independent of hippocampal neurogenesis. J Pharmacol Exp Ther 2007;321: 237-48.

David DJ, Samuels BA, Rainer Q, Wang JW, Marsteller D, Mendez I, et al. Neurogenesis-dependent and -independent effects of fluoxetine in an animal model of anxiety/depression. Neuron 2009;62:479-93.

Detke MJ, Johnson J, Lucki I. Acute and chronic antidepressant drug treatment in the rat forced swimming test model of depression. Exp Clin Psychopharm 1997;5: $107-12$.

Dulawa SC, Holick KA, Gundersen B, Hen R. Effects of chronic fluoxetine in animal models of anxiety and depression. Neuropsychopharmacol 2004;29:1321-30.

Eisch AJ, Petrik D. Depression and hippocampal neurogenesis: a road to remission? Science 2012;338:72-5.

Frankland PW. Neurogenic evangelism: comment on Urbach et al. (2013). Behav Neurosci 2013;127:126-9.

Garthe A, Huang Z, Kaczmarek L, Filipkowski RK, Kempermann G. Not all water mazes are created equal: cyclin D2 knockout mice with constitutively suppressed adult hippocampal neurogenesis do show specific spatial learning deficits. Genes Brain Behav 2014;13:357-64.

Garza JC, Guo M, Zhang W, Lu XY. Leptin restores adult hippocampal neurogenesis in a chronic unpredictable stress model of depression and reverses glucocorticoid-induced inhibition of GSK-3beta/beta-catenin signaling. Mol Psychiatr 2012;17:790-808.

Holick KA, Lee DC, Hen R, Dulawa SC. Behavioral effects of chronic fluoxetine in $\mathrm{BALB} / \mathrm{CJ}$ mice do not require adult hippocampal neurogenesis or the serotonin 1A receptor. Neuropsychopharmacology 2008;33:406-17.

Jaholkowski P, Kiryk A, Jedynak P, Ben Abdallah NM, Knapska E, Kowalczyk A, et al. New hippocampal neurons are not obligatory for memory formation; cyclin D2 
knockout mice with no adult brain neurogenesis show learning. Learn Mem 2009; 16:439-51.

Jaholkowski P, Mierzejewski P, Zatorski P, Scinska A, Sienkiewicz-Jarosz H Kaczmarek L, et al. Increased ethanol intake and preference in cyclin D2 knockout mice. Genes Brain Behav 2011;10:551-6.

Jedynak P, Jaholkowski P, Wozniak G, Sandi C, Kaczmarek L, Filipkowski RK. Lack of cyclin D2 impairing adult brain neurogenesis alters hippocampal-dependent behavioral tasks without reducing learning ability. Behav Brain Res 2012;227: 159-66.

Jiang B, Xiong Z, Yang J, Wang W, Wang Y, Hu ZL, et al. Antidepressant-like effects of ginsenoside Rg1 are due to activation of the BDNF signalling pathway and neurogenesis in the hippocampus. Brit J Pharmacol 2012;166:1872-87.

Kokoeva MV, Yin H, Flier JS. Evidence for constitutive neural cell proliferation in the adult murine hypothalamus. J Comp Neurol 2007;505:209-20.

Kowalczyk A, Filipkowski RK, Rylski M, Wilczynski GM, Konopacki FA, Jaworski J, et al. The critical role of cyclin D2 in adult neurogenesis. J Cell Biol 2004;167: 209-13.

Krishnan V, Nestler EJ. Animal models of depression: molecular perspectives. Curr Top Behav Neurosci 2011;7:121-47.

Lin YL, Wang S. Prenatal lipopolysaccharide exposure increases depression-like behaviors and reduces hippocampal neurogenesis in adult rats. Behav Brain Res 2014;259:24-34.

Liu Q, Yu J, Mao-Ying QL, Mi WL, Li B, Wang YQ et al. Repeated clomipramine treatment reversed the inhibition of cell proliferation in adult hippocampus induced by chronic unpredictable stress. Pharmacogenomics J 2008;8:375-83.

Lu M, Yang JZ, Geng F, Ding JH, Hu G. Iptakalim confers an antidepressant effect in a chronic mild stress model of depression through regulating neuroinflammation and neurogenesis. Int J Neuropsychopharmacol 2014 Mar;13: 1-10 [Epub ahead of print].

McEwen BS, Eiland L, Hunter RG, Miller MM. Stress and anxiety: structural plasticity and epigenetic regulation as a consequence of stress. Neuropharmacology 2012;62:3-12

Mendez-David I, David DJ, Darcet F, Wu MV, Kerdine-Romer S, Gardier AM, et al. Rapid anxiolytic effects of a 5-HT4 receptor agonist are mediated by a neurogenesis-independent mechanism. Neuropsychopharmacology 2014;39: 1366-78.

Meshi D, Drew MR, Saxe M, Ansorge MS, David D, Santarelli L, et al. Hippocampa neurogenesis is not required for behavioral effects of environmental enrichment. Nat Neurosci 2006;9:729-31.

Nollet M, Gaillard P, Tanti A, Girault V, Belzung C, Leman S. Neurogenesis-independent antidepressant-like effects on behavior and stress axis response of a dual orexin receptor antagonist in a rodent model of depression. Neuropsychopharmacology 2012;37:2210-21.

Onksen JL, Brown EJ, Blendy JA. Selective deletion of a cell cycle checkpoint kinase (ATR) reduces neurogenesis and alters responses in rodent models of behavioral affect. Neuropsychopharmacology 2011;36:960-9.

Patricio P, Mateus-Pinheiro A, Sousa N, Pinto L. Re-cycling paradigms: cell cycle regulation in adult hippocampal neurogenesis and implications for depression. Mol Neurobiol 2013;48:84-96.

Perera TD, Dwork AJ, Keegan KA, Thirumangalakudi L, Lipira CM, Joyce N, et al Necessity of hippocampal neurogenesis for the therapeutic action of antidepressants in adult nonhuman primates. PloS One 2011;6:e17600.
Petit-Demouliere B, Chenu F, Bourin M. Forced swimming test in mice: a review of antidepressant activity. Psychopharmacol 2005;177:245-55.

Porsolt RD, Bertin A, Jalfre M. Behavioral despair in mice: a primary screening test for antidepressants. Arch Int Pharmacodyn Ther 1977a;229:327-36.

Porsolt RD, Le Pichon M, Jalfre M. Depression: a new animal model sensitive to antidepressant treatments. Nature 1977b;266:730-2.

Sahay A, Hen R. Adult hippocampal neurogenesis in depression. Nat Neurosci 2007; 10:1110-5.

Sahay A, Scobie KN, Hill AS, O'Carroll CM, Kheirbek MA, Burghardt NS, et al. Increasing adult hippocampal neurogenesis is sufficient to improve pattern separation. Nature 2011;472:466-70.

Samuels BA, Hen R. Neurogenesis and affective disorders. Eur J Neurosci 2011;33: 1152-9.

Santarelli L, Saxe M, Gross C, Surget A, Battaglia F, Dulawa S, et al. Requirement of hippocampal neurogenesis for the behavioral effects of antidepressants. Science 2003:301:805-9.

Schmidt HD, Duman RS. Peripheral BDNF produces antidepressant-like effects in cellular and behavioral models. Neuropsychopharmacology 2010;35:2378-91.

Silva R, Mesquita AR, Bessa J, Sousa JC, Sotiropoulos I, Leao P, et al. Lithium blocks stress-induced changes in depressive-like behavior and hippocampal cell fate: the role of glycogen-synthase-kinase-3beta. Neuroscience 2008;152:656-69.

Singer BH, Jutkiewicz EM, Fuller CL, Lichtenwalner RJ, Zhang H, Velander AJ, et al. Conditional ablation and recovery of forebrain neurogenesis in the mouse. J Comp Neurol 2009;514:567-82.

Solano JM, Jacobson L. Glucocorticoids reverse leptin effects on food intake and body fat in mice without increasing NPY mRNA. Am J Physiol 1999;277:E708-16.

Steru L, Chermat R, Thierry B, Simon P. The tail suspension test: a new method for screening antidepressants in mice. Psychopharmacology 1985;85:367-70.

Surget A, Saxe M, Leman S, Ibarguen-Vargas Y, Chalon S, Griebel G, et al. Drugdependent requirement of hippocampal neurogenesis in a model of depression and of antidepressant reversal. Biol Psychiat 2008;64:293-301.

Udo H, Yoshida Y, Kino T, Ohnuki K, Mizunoya W, Mukuda T, et al. Enhanced adult neurogenesis and angiogenesis and altered affective behaviors in mice overexpressing vascular endothelial growth factor 120. J Neurosci 2008;28: $14522-36$.

Urbach A, Robakiewicz I, Baum E, Kaczmarek L, Witte OW, Filipkowski RK. Cyclin D2 knockout mice with depleted adult neurogenesis learn Barnes maze task. Behav Neurosci 2013;127:1-8.

Wainwright SR, Lieblich SE, Galea LA. Hypogonadism predisposes males to the development of behavioural and neuroplastic depressive phenotypes. Psychoneuroendocrinology 2011;36:1327-41.

Wang Y, Cui XL, Liu YF, Gao F, Wei D, Li XW, et al. LPS inhibits the effects of fluoxetine on depression-like behavior and hippocampal neurogenesis in rats. Prog Neuropsychopharmacol Biol Psychiatry 2011;35:1831-5.

Warner-Schmidt JL, Duman RS. Hippocampal neurogenesis: opposing effects of stress and antidepressant treatment. Hippocampus 2006;16:239-49.

Willner P. Chronic mild stress (CMS) revisited: consistency and behaviouralneurobiological concordance in the effects of CMS. Neuropsychobiology 2005;52:90-110.

Zhu XH, Yan HC, Zhang J, Qu HD, Qiu XS, Chen L, et al. Intermittent hypoxia promotes hippocampal neurogenesis and produces antidepressant-like effects in adult rats. J Neurosci 2010;30:126-63. 